\title{
RECURSOS DIGITAIS EM EDUCAÇÃO: ESTUDO DE CASO DE UM MESTRADO BLENDED EM PORTUGAL
}

\author{
SÃO PAULO/SP JUNHO/2019 \\ $\begin{array}{cc}\text { JOÃO AUGUSTO MATTAR NETO } & \text { - UNINTER \& PUC-SP - joaomattar@gmail.com } \\ \text { DANIELA KARINE RAMOS } & \text { - UFSC - dadaniela@gmail.com } \\ \text { ANA CRISTINA DE CASTRO LOUREIRO } & \text { - IPS - ana.loureiro@ese.jpsantarem.pt }\end{array}$
}

Tipo: Investigação Científica (IC)

Natureza: Relatório Final de Pesquisa

Categoria: Métodos e Tecnologias

Setor Educacional: EDUCAÇÃO SUPERIOR

\begin{abstract}
RESUMO
ESTE ARTIGO INVESTIGA O BLENDED LEARNING EM UM CURSO DE PÓS-GRADUAÇÃO STRICTO SENSU. É UM ESTUDO DE CASO EXPLORATÓRIO. SEU OBJETIVO É DESCREVER A ORGANIZAÇÃO PEDAGÓGICA DE UM MESTRADO BLENDED OFERECIDO EM PORTUGAL E ANALISAR SUAS CONTRIBUIÇÕES PARA A FORMAÇÃO DOS ALUNOS. A METODOLOGIA EMPREGA MÉTODOS DE ANÁLISE QUALITATIVOS E QUANTITATIVOS, UTILIZANDO-SE DA CODIFICAÇÃO E CATEGORIZAÇÃO A PARTIR DAS ORIENTAÇÕES DA ANÁLISE DE CONTEÚDO. UTILIZA COMO INSTRUMENTOS PARA COLETA DE DADOS: ANÁLISE DOCUMENTAL, OBSERVACÃO PARTICIPANTE E UM QUESTIONÁRIO COM PERGUNTAS ABERTAS E FECHADAS. OS RESULTADOS MOSTRAM UMA CONTRADIÇÃO ENTRE A FLEXIBILIDADE DE TEMPO PARA O ESTUDO QUE O BLENDED LEARNING POSSIBILITA E UMA DEMANDA POR MAIS ENCONTROS PRESENCIAIS POR PARTE DOS ALUNOS. O ARTIGO CONCLUI QUE HÁ UM DESAFIO NO EQUILÍBRIO PROPOSTO NO BLENDED LEARNING ENTRE O ESTUDO A DISTÂNCIA E PRESENCIAL.
\end{abstract}

Palavras-chave: BLENDED LEARNING. PÓS-GRADUAÇÃO. EDUCAÇÃO A DISTÂNCIA. 


\section{INTRODUÇÃO}

Este trabalho é um estudo de caso sobre a utilização do blended learning em disciplinas de um curso de mestrado oferecido em Portugal. A questão que move a pesquisa é: como a metodologia do blended learning pode ser estruturada para colaborar com a aprendizagem dos alunos. Seu objetivo é descrever a organização pedagógica desse curso e analisar suas contribuições para formação dos alunos.

A próxima seção discute o conceito de blended learning e sua utilização no ensino superior no Brasil e em Portugal. A terceira seção descreve a metodologia do estudo de caso. A seção seguinte apresenta os resultados da pesquisa, discutidos na quinta seção. A conclusão ressalta as contribuições e limitações do estudo e aponta para trabalhos futuros.

\section{REFERENCIAL TEÓRICO}

\subsection{Blended Learning}

Blended learning (ou aprendizagem híbrida) pode ser definido como a mistura entre a educação presencial e a distância (mais especificamente online). Esta é, por exemplo, a definição de Horn e Staker (2015, p. 44, grifos nossos):

\footnotetext{
O ensino híbrido é um programa de educação formal no qual um estudante aprende, pelo menos em parte, por meio de aprendizagem on-line, sobre o qual tem algum controle em relação ao tempo, ao lugar, ao caminho e/ou ao ritmo e, pelo menos em parte, em um local físico, supervisionado, longe de casa.
}

Mas essa combinação entre presencial/online pode ocorrer de diferentes maneiras. Os autores diferenciam quatro modelos. O modelo de rotação pode envolver rotações por estações, laboratório rotacional, salas de aula invertidas e rotação individual. No modelo à la carte, os alunos cursam algumas disciplinas presenciais e uma ou mais disciplinas online. O modelo flex envolve complementos de atividades presenciais apoiados ou conduzidos, por exemplo, por tutores. Já no modelo virtual enriquecido, são oferecidas algumas sessões presenciais obrigatórias, mas o aluno pode realizar o resto do trabalho online.

No blended learning, os tempos passados em espaço físico de sala de aula são menores - "reduced seat time" (DZIUBAN; HARTMAN; MOSKAL, 2004, p. 2). O blended learning combina, assim, as oportunidades de eficácia e socialização proporcionadas pela sala de aula, com as possibilidades de aprendizagem facilitadas pelas tecnologias e pelos ambientes online (DZIUBAN; HARTMAN; MOSKAL, 2004, p. 
3). Os contextos de aprendizagem em blended learning desenvolvem também, naturalmente, as competências de literacia digital, informacional e científica, fundamentais na sociedade de hoje.

\subsection{Blended Learning no Brasil}

Do ponto de vista legal, não existe blended learning no ensino superior brasileiro: um curso é presencial ou a distância. As instituições de ensino superior podem oferecer, em cursos de graduação presenciais, disciplinas na modalidade a distância até o limite de $40 \%$ da carga horária total do curso, sendo que, para os cursos da área de saúde e das engenharias, o limite é de $20 \%$. De qualquer maneira, esses cursos continuam sendo considerados presenciais, além de que as avaliações e as atividades práticas exigidas nessas disciplinas devem ser realizadas presencialmente (BRASIL, 2018). Acima desse limite, os cursos passam a ser legalmente considerados a distância.

Já os cursos de mestrado e doutorado só podiam até hoje ser oferecidos presencialmente no Brasil. A Portaria Capes no 90 (BRASIL, 2019), entretanto, regulamentou os programas de pós-graduação stricto sensu na modalidade a distância.

\subsection{Blended Learning em Portugal}

O sistema educacional português toma como referência o Processo de Bolonha, que estabeleceu um novo sistema europeu de educação superior (LIMA; AZEVEDO; CATANI, 2008). Esse processo tem como prerrogativa a promoção da mobilidade, do intercâmbio e da empregabilidade dos cidadãos, por meio da maior compatibilidade nos processos formativos, pautando-se em currículos, critérios e metodologias passíveis de comparação (DECLARAÇÃO de Bolonha, 1999). Um dos objetivos centrais foi estabelecer uma estrutura comum de graus facilmente legíveis e comparáveis no ensino superior europeu. Para esse propósito, os países modificaram seus sistemas nacionais para uma estrutura de dois ciclos: $1 . .0$ ciclo (undergraduate) e $2 . .$. ciclo (graduate). $01 .$. ciclo leva a uma qualificação em muitos países denominada "Bacharel" ou "Licenciado", e seus programas têm tipicamente a duração de três anos (seis semestres). Já o 2.ำ ciclo leva a uma qualificação em muitos países denominada "Mestre".

Não existe ainda uma regulamentação quanto ao funcionamento dos cursos superiores na modalidade de b/e-learning em Portugal. Na verdade, no ano de 2019 está sendo discutida em Portugal a regulamentação da educação a distância. De acordo com a legislação em vigor (PORTUGAL, 2016), entretanto, quando da submissão de um novo 
curso à Agência de Avaliação e Acreditação do Ensino Superior (A3ES), deve constar expressamente que o mesmo será ministrado "à distância". Entretanto, apesar de ainda não estar regulamentada, a oferta de ciclos de estudos conferentes de grau na modalidade e/blearning ocorre tanto em Universidades como em Institutos Politécnicos. No momento, é da responsabilidade de cada instituição a definição do desenho curricular e do modelo pedagógico a aplicar a cada curso acreditado.

\section{METODOLOGIA}

Este é um estudo de caso exploratório (YIN, 2015) que utilizou métodos de análise mistos (qualitativos e quantitativos). O contexto de pesquisa foi a primeira oferta do curso de mestrado blended em Recursos Digitais em Educação pelo Instituto Politécnico de Santarém (Portugal). Atualmente, frequentam regularmente o curso 14 alunos, sendo que 12 participaram da pesquisa.

A amostra do estudo compôs-se por conveniência, a partir do aceite dos alunos para responder a um questionário. Em relação à dispersão geográfica, seis alunos residem em Santarém, cidade em que fica a sede do curso, e seis residem em outras cidades. Ainda em relação ao perfil, dez alunos exercem atividade profissional, sete deles atuando como professores. Metade dos alunos que exerce atividade profissional dedica de 30 a 40 horas semanais à atividade laboral, sendo que $25 \%$ dos alunos dedica mais de 40 horas de sua carga horária semanal ao trabalho.

Além da análise documental do curso e de um questionário (https://bit.ly/2Xdh0Zu) enviado aos alunos, com perguntas abertas e fechadas, foi utilizada a metodologia da observação participante. Os dados numéricos foram tabulados e analisados estatisticamente, enquanto os dados qualitativos foram codificados e categorizados seguindo as orientações da análise de conteúdo (BARDIN, 2016).

\section{RESULTADOS}

\subsection{Projeto e Oferta do Curso}

O curso Recursos Digitais em Educação é ofertado pelo Instituto Politécnico de Santarém como blended e sua primeira turma está em andamento. Foi acreditado pelo Conselho de Administração da Agência de Avaliação e Acreditação do Ensino Superior de Portugal em 2018. As suas unidades curriculares utilizam a metodologia blended com duração de quatro semestres, contabilizando uma carga horária de 420 horas de contato (mais 72 horas de orientação tutorial), em um total de 120 ECTS (European Credit 
Transfer Scale). Sua finalidade é "construir e aprofundar, com os agentes educativos, competências na utilização, avaliação, concepção e desenvolvimento de recursos pedagógicos digitais numa perspectiva de inovação e investigação educacional". Ampliam-se os objetivos gerais para o desenvolvimento de competências digitais e pedagógicas relacionadas à integração das TIC (Tecnologias da Informação e da Comunicação) para a promoção de prática inovadoras, o incremento de metodologias de aprendizagem ativa e melhoria das aprendizagens (INSTITUTO POLITÉCNICO DE SANTARÉM, 2018).

A partir desse objetivo geral, descrevem-se os objetivos de aprendizagem a serem atingindo em diferentes contextos educativos, de modo a contemplar conhecimentos, aptidões e competências a serem desenvolvidas pelos estudantes. São eles:

a. analisar e compreender a problemática e os desafios da integração das TIC;

b. selecionar, avaliar e usar, de forma sustentada e criativa, RED (Recursos Educativos Digitais);

c. conceber RED capazes de responder às necessidades da comunidade educativa;

d. planeJar e implementar estratégias, metodologias e práticas de ensino inovadoras e inclusivas com recurso às TIC;

e. elaborar e integrar projetos de desenvolvimento e práticas investigativas para a construção de comunidades inclusivas e digitalmente literadas;

f. refletir criticamente sobre as práticas de ensino e aprendizagem numa perspetiva de melhoria da ação profissional;

g. reconhecer a importância da formação contínua e ao longo da vida no domínio da tecnologia educativa. (INSTITUTO POLITÉCNICO DE SANTARÉM, 2018).

Os planos das unidades curriculares que compõem o curso descrevem as metodologias a serem desenvolvidas, dentre as quais destacam-se:

a. aulas teórico-práticas com exposição-participação;

b. pesquisa de informação on-line para aprofundamento dos conhecimentos;

c. análise e discussão crítica de documentos;

d. análise e reflexão crítica de modelos e práticas;

e. exploração de ferramentas e recursos digitais;

f. construção coletivas de propostas de intervenção;

g. participação em fóruns de discussão;

h. análise e discussão de casos;

i. exercícios de construção de textos. 
O Mestrado utiliza um ambiente virtual de aprendizagem (AVA) que apresenta características específicas, como o fato de ser um espaço de informação e socialização, em que os estudantes não são apenas ativos, mas também atores, integrando múltiplas ferramentas que se complementam. Assim, o espaço virtual primordial de interação e partilha de conteúdos e consulta de informação é o Moodle. Com o intuito de fortalecer o sentido de comunidade entre os participantes do curso, o AVA é complementado com outros espaços virtuais, a saber: grupo no WhatsApp e grupo no Skype (conversação mais imediata), sala de videoconferências Colibri-Zoom (sessões síncronas online), grupo no Facebook (partilha de notícias, eventos, informação relevante para o curso) e grupo no Mendeley (curadoria de conteúdos, repositório de recursos científicos). Esses espaços virtuais sociais educacionais fomentam a capacidade de colaboração entre os participantes, tanto de forma síncrona como assíncrona; de criação de um perfil pessoal construído apenas em torno das especialidades e dos interesses educacionais e curriculares, tornando mais fácil encontrar outras pessoas, recursos, eventos e discussões em torno dos mesmos interesses; e de mais facilmente encontrar, organizar, gerir e partilhar informações e conteúdos.

Com o intuito de enriquecer o curso e proporcionar aos estudantes a possibilidade de contatar com diferentes especialistas, nas sessões presenciais têm sido convidados especialistas para Seminários, Workshops e Palestras sobre diversas temáticas na área dos Recursos Digitais em Educação. Essas sessões são interativas, colaborativas, atrativas e ativas, em que os estudantes têm a oportunidade de interagir com os especialistas convidados, questionando, partilhando e construindo conhecimentos a partir das informações veiculadas e dos recursos educativos explorados.

Tal como Palloff e Pratt (2005), considera-se que os principais benefícios de uma aprendizagem colaborativa são: o desenvolvimento da capacidade de pensamento crítico, a co-criação de conhecimento e de significado, a reflexão e a aprendizagem transformativa.

Vale notar que as sessões presenciais são abertas à comunidade, partindo-se da premissa de que partilhar é, provavelmente, a característica mais básica da educação: a educação é partilhar conhecimentos, ideias e informações com os outros, construindo novos conhecimentos, habilidades, ideias e compreensão. Defende-se que todos os cidadãos devem ter acesso a experiências e recursos educacionais de alta qualidade, contribuindo assim para esse bem comum e para uma sociedade mais informada, baseada no conhecimento.

\subsection{Análise das Respostas ao Questionário}


No que se refere aos hábitos de estudo dos alunos no curso, observa-se que a maioria dedica entre 3 e 5 horas por semana para estudar, conforme o Gráfico 1.

Gráfico 1 - Horas semanais dedicadas aos estudos

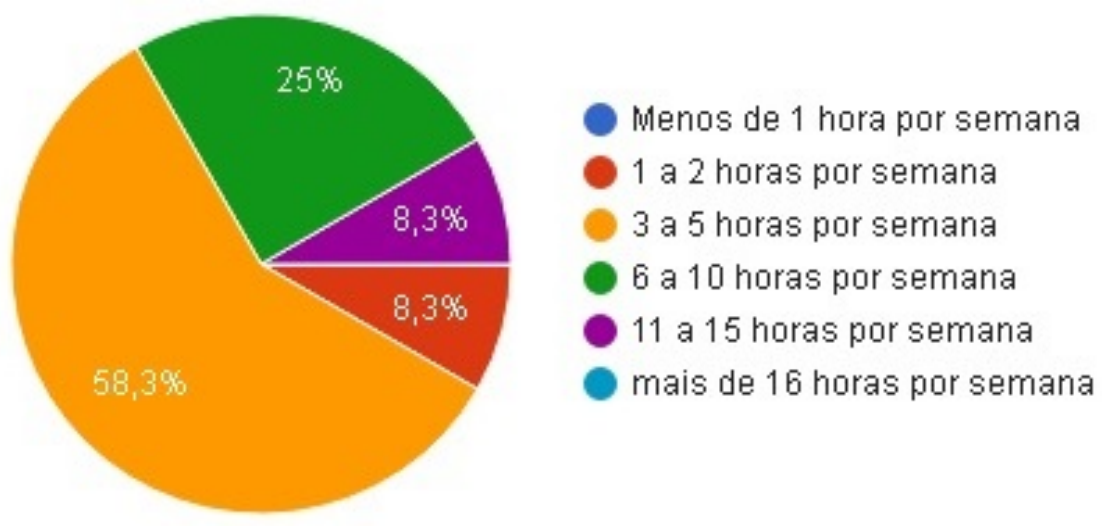

Fonte: os autores, a partir da compilação das respostas ao questionário

Ainda sobre a relação com os estudos, quando questionados sobre a principal dificuldade para estudar, $75 \%$ dos alunos apontaram a falta de tempo.

Quando os estudantes foram questionados sobre qual dos vários aspectos que compõem o curso blended colaboram mais efetivamente para a sua aprendizagem, destacou-se a interação mediada com os colegas e professores $(33,3 \%)$, seguida pela interação presencial (25\%) e, na sequência, as atividades e os estudos realizados a distância (16,7\%). Destaca-se ainda que 10 alunos concordam plenamente que estão tendo uma aprendizagem significativa no curso; 9 concordam plenamente e 2 concordam que interagem com colegas e professores; e 9 concordam plenamente que participam motivados.

Quando os alunos completaram as frases "O que mais gosto no curso por ser blended", destacou-se a flexibilidade espaço-temporal, especialmente a possibilidade de realizar pesquisas em qualquer momento do dia, conseguir organizar o estudo em períodos em que se tem maior disposição para aprender e conciliar melhor o tempo com outras atividades laborais e pessoais.

Em relação ao uso das TIC, os alunos destacaram a possibilidade de conhecer e explorar diferentes recursos tecnológicos, o favorecimento à utilização de diversificados recursos e materiais no processo de aprendizagem, o desenvolvimento de capacidades técnicas relacionadas ao uso das tecnologias no processo de ensino e aprendizagem, a contribuição para se sentirem mais preparados para sua futura integração na prática 
pedagógica, que os materiais e as atividades apresentam-se mais organizados e tem-se o registro da trajetória da aprendizagem

Em relação a "O que menos gosto no curso por ser blended", destacou-se a demanda por mais encontros presenciais, que favorecem maior integração com os colegas e professores, apoiam a organização das atividades que são realizadas a distância, permitem sentir-se mais parte de um grupo e de uma instituição e reforçar e aprofundar os conteúdos estudados a distância, e diversificam as possibilidades de aprendizagem e as formas de avaliação

\section{DISCUSSÃO}

Cabe inicialmente destacar que o modelo de blended learning do mestrado estudado não é à la carte, como ocorre em praticamente todos os cursos de graduação no Brasil, em que uma ou algumas disciplinas são oferecidas totalmente a distância, mas um modelo virtual enriquecido, em que parte das atividades do curso é oferecida presencialmente, mas a maior parte a distância. Isso desafia a dicotomia presencial/distância que marca a legislação e a prática pedagógica brasileiras, e, apesar de a educação a distância somente em 2019 estar em fase de regulamentação em Portugal, posiciona o curso em um patamar que apenas agora a legislação brasileira começa a visitar. Todavia, cabe ressaltar que os mestrados em Portugal (e na Comunidade Europeia em geral) têm uma configuração distinta dos mestrados no Brasil, em função do Processo de Bolonha.

O perfil dos alunos do Mestrado traz informações importantes para a discussão. Em primeiro lugar, metade reside em outra cidade, o que talvez não fosse possível no caso de a oferta ser presencial. Além disso, o trabalho ocupa de 30 a 40 horas do seu tempo, o que também provavelmente inviabilizaria a participação em um curso presencial. Como Dziuban, Hartman e Moskal (2004) afirmam, no blended learning o tempo passado em sala de aula é naturalmente menor. Nesse sentido, como pudemos observar, o projeto do curso prevê uma série de atividades a distância, o uso de diversas ferramentas além do AVA, diferentes metodologias e atividades colaborativas.

Entretanto, nas respostas ao questionário foi possível observar uma contradição entre a flexibilidade temporal possibilitada pelo blended learning e a demanda dos alunos por mais encontros presenciais. Apenas $25 \%$ dos alunos apontaram que a interação presencial era um aspecto do blended learning que contribuía para sua aprendizagem, mas boa parte dos alunos indicou a preferência por mais encontros presenciais. Como a maior parte dos alunos reclama de falta de tempo para estudar (dedicando uma média 
de 4 horas ao estudo por semana), essa demanda soa paradoxal, tanto porque exigiria mais tempo dos alunos, quanto porque indica uma dificuldade de gestão do tempo de estudo a distância, apesar de a flexibilidade temporal ter sido indicada como o aspecto mais positivo do blended learning para a aprendizagem.

Foi ainda possível perceber que as TIC desempenham um papel importante no blended learning para os alunos. Como observamos, o próprio projeto do curso aponta para o objetivo de desenvolver competências digitais e pedagógicas relacionadas à sua integração para a promoção de práticas inovadoras e contribuir para a aprendizagem.

Deve-se ressaltar ainda o caráter de abertura do curso à comunidade, contribuindo assim para um dos pilares do ensino universitário, a extensão.

\section{CONSIDERAÇÕES FINAIS}

Este artigo apresentou e discutiu os resultados de um estudo de caso em um curso de mestrado em Portugal que utiliza a metodologia blended learning. Nesse sentido, contribuiu para apresentar o projeto e as características de um curso de pós-graduação stricto sensu ofertado nessa modalidade.

Ressalta-se que os alunos avaliaram positivamente o curso no formato blended. Nesse sentido, este trabalho contribui também para a literatura que avalia os resultados do blended learning, mais especificamente em curso de mestrado.

Uma das limitações deste estudo é a amostra pequena. Nesse sentido, trabalhos futuros poderiam replicar a metodologia de análise, comparando inclusive cursos no Brasil com outros oferecidos em Portugal.

\section{REFERÊNCIAS}

BARDIN, Laurence. Análise de conteúdo. São Paulo: Almedina, 2016.

BRASIL. Ministério da Educação (MEC). Fundação Coordenação de Aperfeiçoamento de Pessoal de Nível Superior - CAPES. Portaria no 90, de 24 de abril de 2019. Dispõe sobre os programas de pós-graduação stricto sensu na modalidade de educação a distância.

BRASIL. Ministério da Educação (MEC). Portaria no 1.428, de 28 de dezembro de 2018. Dispõe sobre a oferta, por Instituições de Educação Superior - IES, de disciplinas na 
modalidade a distância em cursos de graduação presencial.

DECLARAÇÃO de Bolonha. Declaração conjunta dos ministros da educação europeus, assinada em Bolonha. 19 jun. 1999. Disponível em: http://www.fam.ulusiada.pt/downloads/bolonha/Docs02_DeclaracaoBolonha.pdf

DZIUBAN, C.; HARTMAN, J.; MOSKAL, P. Blended Learning. Educause Center for Applied Research. Research Bulletin, v. 2004, n. 7, 30 mar. 2004.

HORN, M. B.; STAKER, H. Blended: usando a inovação disruptiva para aprimorar a educação. Trad. Maria Cristina Gularte Monteiro. Porto Alegre: Penso, 2015.

INSTITUTO POLITÉCNICO DE SANTARÉM. Escola Superior de Educação. Recursos Digitais em Educação. Disponível em: https://siese.ipsantarem.pt/ese/cursos_geral.FormView?P_CUR_SIGLA=MRDE. Acesso em: 30 abr. 2019.

LIMA, Licínio C.; AZEVEDO, Mário Luiz Neves de; CATANI, Afrânio Mendes. O processo de Bolonha, a avaliação da educação superior e algumas considerações sobre a Universidade Nova. Avaliação: Revista da Avaliação da Educação Superior, v. 13, p. 7-37, 2008.

OPEN Education Consortium. Disponível em: https://www.oeconsortium.org/. Acesso em: 12 maio 2019.

PALLOFF, R. M.; PRATT, K. Collaborating online: learning together in community. San Francisco, CA: Jossey-Bass, 2005.

PORTUGAL. Ministério da Ciência, Tecnologia e Ensino Superior. Decreto-Lei oㅡ 74/2006, de 24 de março de 2006. Diploma que aprova o regime jurídico dos graus e diplomas do Ensino Superior. Diário da República, I Série-A, n. 60, p. 2242-2257, 24 mar. 2006.

YIN, R. K. Estudo de caso: planejamento e métodos. 5. ed. Porto Alegre: Bookman, 2015 . 